\title{
ASCUS Negative Conversion in Pelvic Floor Reconstruction of POP Patients with Human Papillomavirus-Negative ASCUS Cytology
}

\section{Ai-Min Wei ( $\sim 769528908 @ q q . c o m$ )}

Nanjing Maternity and Child Health Care Hospital https://orcid.org/0000-0002-6118-7695

\section{Yun Fan}

Nanjing Medical University

\section{Yu-Fei Shen}

Nanjing Maternity and Child Health Care Hospital

Lei Zhang

Nanjing Maternity and Child Health Care Hospital

Xiao-Mei Tan

Nanjing Maternity and Child Health Care Hospital

\section{Research article}

Keywords: Pelvic organ prolapse, Pelvic floor reconstruction, Pap test, Atypical squamous cells of undetermined significance, Negative conversion

Posted Date: March 31st, 2020

DOl: https://doi.org/10.21203/rs.3.rs-20040/v1

License: (c) (1) This work is licensed under a Creative Commons Attribution 4.0 International License. Read Full License 


\section{Abstract}

Objective

To inquire into the influence of the pelvic floor reconstruction surgery of pelvic organ prolapse on Papillomavirus-Negative atypical squamous cells of undetermined significance cytology.

Methods

This investigation was studied retrospectively about female patients of POP 3 4 stage with high-risk human papillomavirus DNA-negative ASCUS between January, 2008 and September, 2018.All patients received one of two treatment regimens, pelvic floor reconstruction or local hydrocortisone cream $(0.1 \%$ Hydrocortisone Butyrate cream, $1 \mathrm{~g}$ nightly for no less than 12 weeks). After 6 months follow-up, we collected data to compare the positive rate of ASCUS with high-risk human papillomavirus DNA-negative between the two groups after intervention.

Results

The negative conversion rate of ASCUS in the operative patients was higher than in patients using local hydrocortisone cream (33/47 [70.2\%] vs 19/44 [43.2\%], P = 0.009).

\section{Conclusions}

The decline of non-human papillomavirus ASCUS cytology in postoperative patients might be related to pelvic floor reconstruction.

\section{Background}

The diagnostic rate of atypical squamous cells of undetermined significance cytology(ASCUS)in cervical cytology screening was $3-10 \%[1,2]$. There were few reports about the percentage of ASCUS in postmenopausal women. It should not be lower than the level of non-menopausal women [3, 4].Explicit screening and treatment guidelines for HPV related cervical lesions can be obtained.[7]However, we noted that there was a lack of clinical data on the prevalence of HPV-negative ASCUS in POP patients.Only one report suggested that $6.6 \%$ of POP patients have abnormal thinprep cytologic test(TCT)[8].As we know,Although chronic inflammation accounted for $40 \%-70.1 \%$ of the pathological results of biopsy in ASCUS, little research has been done on the causes of HPV-negative cervical cytology ASCUS in POP.Not long ago one study from Kimia Menhaji's suggested HPV-negative cytological abnormalities might be associated with POP. They reminded gynecologists who hoped to retain cervix in women with POP to draw a certain degree of attention.[8] However, the influence of surgery on human papillomavirus negative thin-layer cytology ASCUS has not been reported. According to Kimia Menhaji's result, POP resulting in Pap smear abnormalities, we hypothesize pelvic floor reconstruction may result in the negative conversion of ASCUS. 
In this study we retrospectively investigated the influence of the surgery on Human negative cytology ASCUS and compared with patients treated with local hydrocortisone cream,lt is well known that hydrocortisone is a kind of corticosteroid and has a certain therapeutic effect on chronic cervical inflammation. $[9,10]$ We tried to assess the effects of pelvic floor reconstruction on ASCUS at six months'postoperation so as to tell us as to which kind of surgery to do with patients who come in for POP with ASCUS pap,This will be helpful for clinicians to make correct choices of therapeutic regimen on POP patients with HPV-negative ASCUS.

\section{Methods}

\section{Study design}

A retrospective cohort study was performed from January ,2008 to September, 2018. at the Department of Obstetrics and Gynecology of Nanjing maternity and child Health Care Hospital.The study was conducted in accordance with the Declaration of Helsinki.The protocol was approved by the Ethics Committee of Obstetrics and Gynecology Hospital of Nanjing Medical University(2019-31).

We collected patients of uterine prolapse (with/without cystocele)that was stage III or IV by POP-Q with human papillomavirus-negative cervical cytology ASCUS, which was confirmed as inflammation by cervical biopsy.The patients were investigated for after 6 months with HPV and Pap tests. Cervical biopsy was performed for ASCUS patients at 6 months'follow-up. All patients hadn't received treatment with estrogen because of ASCUS. The treatment of women in the hydrocortisone group was enquired in detail and the patients who completed the treatment for no less than 12 weeks were included in the survey. All women were included in the study if they treated with local hydrocortisone cream or received operation treatment between January ,2008 and September, 2018.

Patients were excluded if there was any of the following conditions: history of a cervical excisional procedure or hysterectomy, history of cervical or vaginal intraepithelial neoplasia ,history of cervical,vaginal, or endometrial cancer,and also exclusion of CIN ,VIN and cancer by transvaginal cervical biopsy before treatment,no HPV results of follow-up, We followed the STROBE guidelines in our retrospective study[11].

Sample size was calculated by $G$ * Power 3.1 software. Natural negative conversion rate of postmenopausal ASCUS was about 23/80, (28.8\%)[12]; The curative ratio of hydrocortisone to the chronic cervicitis is $0 \%[10]$.so the negative conversion rate of ASCUS in hydrocortisone group was about $30 \%$; The negative conversion rate of operation wasn't reported,according to our pre-experiment, we set P1 to $0.65, \mathrm{P} 2=0.30$;Other parameters are tail: two; alpha level to 0.05 ; power to $0.90 ; \mathrm{N} 1 / \mathrm{N} 2=1$. A sample size of 91 patients in total groups should be surveyed to detect statistically significant differences allowed $10 \%$ follow-up loss.

\section{Intervention}


A retrospective cohort study was done, comparing two groups: patients treated with pelvic floor reconstruction $(n=47)$ or local hydrocortisone cream $(n=44)$. The review of medical records were blinded. The surgery of pelvic floor reconstruction with transvaginal mesh was performed by three surgeons with senior professional titles. The standardized operation procedures were carried out from the beginning of the study.The control group was non-operative scheme, in which patients were applied $0.1 \%$ hydrocortisone butyrate cream nightly for 12 weeks,As we know,Hydrocortisone is a mild corticosteroid with anti-inflammatory effects on these diseases in which inflammation is a prominent feature.

\section{Outcomes}

We collected the imformation of Pap tests/ HPV tests and Colposcopy examinations and biopsy by reviewing the electronic medical record.Pap tests were reported according to the 2001 Bethesda System. HPV tests were tested by HybridCapture 2 (Digene Corporation) or DNA flow-through hybridization genotyping technique according to the manufacturer's instructions. (reference range was $0 \sim 1 \mathrm{pg} / \mathrm{ml}$ ) .Cervical biopsy was performed in patients with continuous ASCUS cytology.

Colposcopy examinations and biopsy were used to exclude cervical or vaginal intraepithelial neoplasia or cancer.

We also gathered patients' clinical characteristics, including age ,body mass index, parity, cesarean ,tobacco use, alcohol use, diabetes, and hypertension, POP-Q stage, concomitant cystocele, local mucosal ulcer, currently sexually active, to perform evaluation.

\section{Statistical analysis.}

Continuous data were described as mean \pm standard deviation (SD). Categorical data and ranked data were reported as count and percent.The two-sample t test was used to compare continuous data between two groups. The pearson chi-square test or Fisher exact test or continuity Correction was used to explore differences between two groups for categorical data. Multivariate logistic regression analysis was applied to assess the variables related to ASCUS negative conversion. We used Wilcoxon rank sum test to compare ranked data between two groups .

Statistical analyses were performed using SPSS19.0 statistical software, All reported P values were 2sided, and $P$ value $<0.05$ was considered statistically significant.

\section{Results}

A total of 44 cases of local hydrocortisone cream group and 47 cases of pelvic floor reconstruction group were included in the statistical analysis. We recorded the baseline characteristics of the two cohorts, The clinical characteristics of the two groups are listed in Tables 1 . Women in the study group were older than patients in the control group $(P=0.028)$. There was no significant difference between the two groups with 
respect to body mass index, parity, cesarean ,tobacco use, alcohol use, diabetes, and hypertension. (each $P>0.05)$.

Table 1

Baseline Clinical Characteristics of two groups

\begin{tabular}{|llll|}
\hline Baseline characteristics & $\begin{array}{l}\text { operation group, } \\
\mathbf{n = 4 7}\end{array}$ & $\begin{array}{l}\text { local hydrocortisone group, } \\
\mathbf{n = 4 4}\end{array}$ & p value \\
\hline Age(years),mean (SD) & $68.6(8.8)$ & $59.0(7.1)$ & $0.028 \mathrm{~b}$ \\
\hline Body mass index (kg/m2),mean (SD) & $25.7(3.8)$ & $23.0(4.9)$ & $0.797 \mathrm{~b}$ \\
\hline Parity(median),mean (SD) & $1.85(0.659)$ & $2.02(0.664)$ & $0.219 \mathrm{~b}$ \\
\hline cesarean (n, \%) & $22(46.8)$ & $16(36.4)$ & $0.313 \mathrm{a}$ \\
\hline Tobacco use, $\%$ & $8(17.0)$ & $9(20.5)$ & $0.675 \mathrm{a}$ \\
\hline Alcohol use, $\mathrm{n}(\%)$ & 27 & 20 & $0.253 \mathrm{a}$ \\
\hline Diabetes (n, \%) & $10(22.7)$ & $9(19.1)$ & $0.675 \mathrm{a}$ \\
\hline Hypertension (n, \%) & $20(42.6)$ & $17(38.7)$ & $0.704 \mathrm{a}$ \\
\hline PoP-Q = Pelvic Organ Prolapse Quantification. & & \\
\hline a.pearson Chi-Square, & & & \\
\hline b.equal variances assumed & & & \\
\hline
\end{tabular}

Results showed that the rate of papillomavirus-Negative ASCUS in the postoperative patients was lower than in local hydrocortisone cream group (13/47 [27.7\%] vs23/44 [52.3\%], $P=0.009)$ (Table 2).Since there was difference in age between the two groups, we furtherly divided the age into three sub-groups to examine whether the factor of age had an impact on the negative conversion rate of ASCUS.It revealled from Table 3 that there was no significant difference in ASCUS negative conversion rate between the two groups in different age sub-groups $(P=0.737)$. 
Table 2

the negative conversion rate of ASCUS in two groups of POP patients with papillomavirus-Negative ASCUS

\begin{tabular}{|llll|}
\hline thin-layer cytology & $\begin{array}{l}\text { local hydrocortisone cream } \\
\text { group, }(\mathbf{n = 4 4 )}\end{array}$ & $\begin{array}{l}\text { operation group, } \\
(\mathbf{n = 4 7 )}\end{array}$ & $\begin{array}{l}\mathbf{p} \\
\text { value }\end{array}$ \\
\hline $\begin{array}{l}\text { Patients with ASCUS negative } \\
\text { conversion , }(\%)\end{array}$ & $19(43.2)$ & $33(70.2)$ & $0.009 a$ \\
\hline a.ASCUS = atypical squamous cell of undetermined signification & & \\
\hline b.pearson Chi-Square & & & \\
\hline
\end{tabular}

Table 3

Comparison of two groups of ASCUS negative conversion patients in different age sub-groups

\begin{tabular}{|lllllll|}
\hline Patients with ASCUS negative conversion , $\mathrm{n}(\%$ & age & & & & p \\
& & $50 \sim$ & $60 \sim$ & $70 \sim$ & total & \\
& $59 \mathrm{y}$ & $69 \mathrm{y}$ & $79 \mathrm{y}$ & & \\
\hline local hydrocortisone cream group , $\mathrm{n}(\%)$ & 4 & 9 & 6 & 19 & $0.737 \mathrm{a}$ \\
operation group, $\mathrm{n}(\%)$ & 9 & 14 & 10 & 33 & \\
\hline Data are presented as \% or the mean \pm SD, unless otherwise indicated. & & & \\
\hline POPQ = Pelvic Organ Prolapse Quantification. & & & & & \\
\hline a Mann-Whitney test. & & & & & \\
\hline b Fisher's exact test. & & & & & \\
\hline
\end{tabular}

We put 5 relevant variables, such as POP-Q stage, concomitant cystocele, local mucosal ulcer, currently sexually active and operation, into multivariate logistic analysis.Logistic regression revealed that pelvic floor reconstruction was the only factor of ASCUS negative conversion rate(Table 4).

Table 4

Multivariate analysis of factors related to ASCUS negative conversion

\begin{tabular}{|lllllll|}
\hline Variables & $\boldsymbol{\beta}$ & S.E, & Wald $\chi 2$ & OR & $95 \%$ Cl for EXP(B) & P \\
\hline operation & 1.182 & 0.459 & 6.638 & 3.259 & $1.327 \sim 8.008$ & 0.010 \\
\hline POP-Q stage & -1.044 & 0.666 & 2.461 & 0.352 & $0.095 \sim 1.297$ & 0.117 \\
\hline Concomitant cystocele & 0.247 & 0.491 & 0.252 & 1.280 & $0.489 \sim 3.352$ & 0.615 \\
\hline local mucosal ulcer & 0.281 & 0.483 & 0.339 & 1.325 & $0.514 \sim 3.411$ & 0.560 \\
\hline Currently sexually active & -0.303 & 0.514 & 0.348 & 0.739 & $0.270 \sim 2.023$ & 0.555 \\
\hline
\end{tabular}


We also observed that one case of HPV-negative ASCUS in operation group developed into cervical intraepithelial neoplasia grade 1 postoperatively.

\section{Discussion}

We found that the negative conversion rate of ASCUS in operation group was significantly higher than that in local hydrocortisone cream group. ASCUS negative conversion had a statistical accosiation with the operation of pelvic floor reconstruction.

Our investigation is the first study on the relationship between pelvic floor reconstruction and ASCUS negative conversion.

The mechanism of abnormal pap smears caused by POP is still unclear. Previous studies have reported on the relationship between vaginal mucosal atrophy and abnormal pap smear [13]. Prolapse is also associated with vaginal epithelial changes similar to atrophy.Badiou [14] has reported the atrophic changes of prolapsed tissues compared with vaginal tissues of non-prolapse.Pelvic organ prolapse is associated with changes in connective tissue structure, including changes in collagen types expression and structural disorders [15].Söderberg[16] has found there was a decrease in the collagen concentration in biopsies of prolapsed women under 53 years of age.

Natural negative conversion rate of postmenopausal ASCUS was about 28.8\%.[12], Our results showed that the operation group had an effect with $70.2 \%$ of negative conversion rate, while $43.2 \%$ of hydrocortisone cream group, which was significantly better than that of the latter $(P=0.009)$. Our study suggests that there is a statistical correlation between ASCUS negative conversion and the surgery of pelvic floor reconstruction.

In our retrospective cohort study, women in the operation group were older than hydrocortisone group,that is to say, age may be a confounding factor.So,in the next article, we furtherly subdivided the patients into three different age subgroup and compared the negative conversion rate of ASCUS between the two groups in different age subgroup distribution $(P=0.737)$, thus we generally excluded the influence of age on intervention measures.

\section{Conclusions}

Many POP patients with HPV-negative ASCUS prefer to receive uterine sparing prolapse surgery when they come for treatment.But the clinicians is in a dilemma. They are worried the further pathological changes of the cervix.

Our study suggested that the POP patients with HPV-negative ASCUS could received pelvic floor reconstruction with uterus in situ after excluding cervical lesions preoperatively by biopsy. With the treatment of prolapse after operation, ASCUS might turn negative incidentally. But we imcompletely 
excluded the possibility of cervical lesions progression in a very small number of patients, which should be paid attention to by clinicians after operation.

Although we controlled for potentially important confounders such as age, sexual life, smoking, and degree of prolapse etc in this trial, Our retrospective study was a single center trial of short follow-up time, which may lead to the bias of the research results.So multicenter epidemiological surveys,and longer time of follow-up can confirm the above findings in our study.Nevertheless, our results provide a reference that will be clinically useful for clinicians to choose operation methods.

\section{Abbreviations}

95\% Cl: 95\% Confidence Interval; SD: Standard Deviation; POP: Pelvic organ prolapse; ASCUS:Atypical squamous cells of undetermined significance

\section{Declarations}

Ethics approval and consent to participate: The protocol was approved by the Ethics Committee of Obstetrics and Gynecology Hospital of Nanjing Medical University. All participants provided written informed consent.

Consent for publication: Not Applicable.

Competing interests: The authors declare that they have no competing interests.

Funding: This research did not receive any specific grant from funding agencies in the public, commercial, or not-for-profit sectors.

Authors' contributions: AMW: Design, Data Collection, Manuscript Preparation

YF : Data Analysis,Statistical Analysis; YYFS ,LZ and XMT performed the surgery. All authors read and approved the final manuscript.

Acknowledgments: Not applicable

Availability of data and materials: The datasets used and analyzed during the current study are available from the corresponding author on reasonable request.

\section{References}

1 Arbyn M , Castellsague X, de Sanjose S, et al. Worldwide burden of cervicalcancer in 2008[J]. Ann Oncol, 2011,22囚12邓:2675-2686. 
2 Kurman RJ, Henson DE, Herbst AL, et al. Interim guidelines for management of abnormal cervical cytology. The 1992 National Cancer Institute Workshop.JAMA. 1994 Jun 15;271(23):1866-9.

3 Behiye Pinar Cilesiz Goksedef, Ozgur Akbayir, Safak Yilmaz Baran .Atypical squamous cells of undetermined significance in postmenopausal women: a comparative retrospective analysisEuropean Journal of Obstetrics \& Gynecology and Reproductive Biologyy 159 (2011) 418-421

4 Flynn K,Rimm DL.Diagnosis of "ASCUS" in women over age 50 is less likely to be associated with dysplasia.Diagn Cytopathol.2001.24(2):132-136

5 Demirtas GS, Akman L, Demirtas O,Clinical significance of ASCUS and ASC-H cytological abnormalities: a six-year experience at a single center.Eur J Gynaecol Oncol. 2015;36(2):150-4.

6 Goksedef BP, Akbayir O, Baran SY区Atypical squamous cells of undetermined significance in postmenopausal women: a comparative retrospective analysis. Eur J Obstet Gynecol Reprod Biol. 2011 Dec;159(2):418-21.

7 ACOG Practice Bulletin. Management of Abnormal Cervical Cytology and Histology Obstetrics \& Gynecology 2008 (112)6:1419-1444

8 Menhaji K, Harvie HS, Cheston E, Relationship Between Pelvic Organ Prolapse and Non-Human Papillomavirus Pap Smear Abnormalities.Female Pelvic Med Reconstr Surg. 2018 Jul/Aug;24(4):315-318

9 Zanoschi Ch, Anton C, Anton E .Cervugid ovules in cervico-vaginal infections and cervix uteri precancerous conditions treatment.Rev Med Chir Soc Med Nat lasi. 2004 Jul-Sep;108(3):628-34.

10 Mattson SK, Polk JP, Nyirjesy P. Chronic Cervicitis: Presenting Features and Response to Therapy. J Low Genit Tract Dis. 2016 Jul;20(3):e30-3.

11 Vandenbroucke JP, von Elm E, Altman DG, et al. Strengthening the Reporting of Observational Studies in Epidemiology (STROBE): explanation and elaboration. Int J Surg 2014;12(12):1500-1524

12 Rader $A E$, Rose $P G$, Rodriguez M $\triangle A$ typical squamous cells of undetermined significance in women over 55. Comparison with the general population and implications for management.Acta Cytol. 1999 May-Jun;43(3):357-62.

13 Tambouret RH, Wilbur DC. The many faces of atrophy in gynecologic cytology. Clin Lab Med 2003;23(3):659-679.

14 Badiou W, Granier G, Bousquet PJ, et al. Comparative histological analysis of anterior vaginal wall in women with pelvic organ prolapse or control subjects. A pilot study. Int Urogynecol J Pelvic Floor Dysfunct 2008;19(5):723-729 
15 Vetuschi A, D'Alfonso A, Sferra R, et al. Changes in muscularis propria of anterior vaginal wall in women with pelvic organ prolapse. Eur J Histochem 2016;60(1):2604

16 Söderberg MW, Falconer C, Byström B, et al. Young women with genital prolapse have a low collagen concentration. Acta Obstet Gynecol Scand 2004;83:1193-1198

\section{Figures}




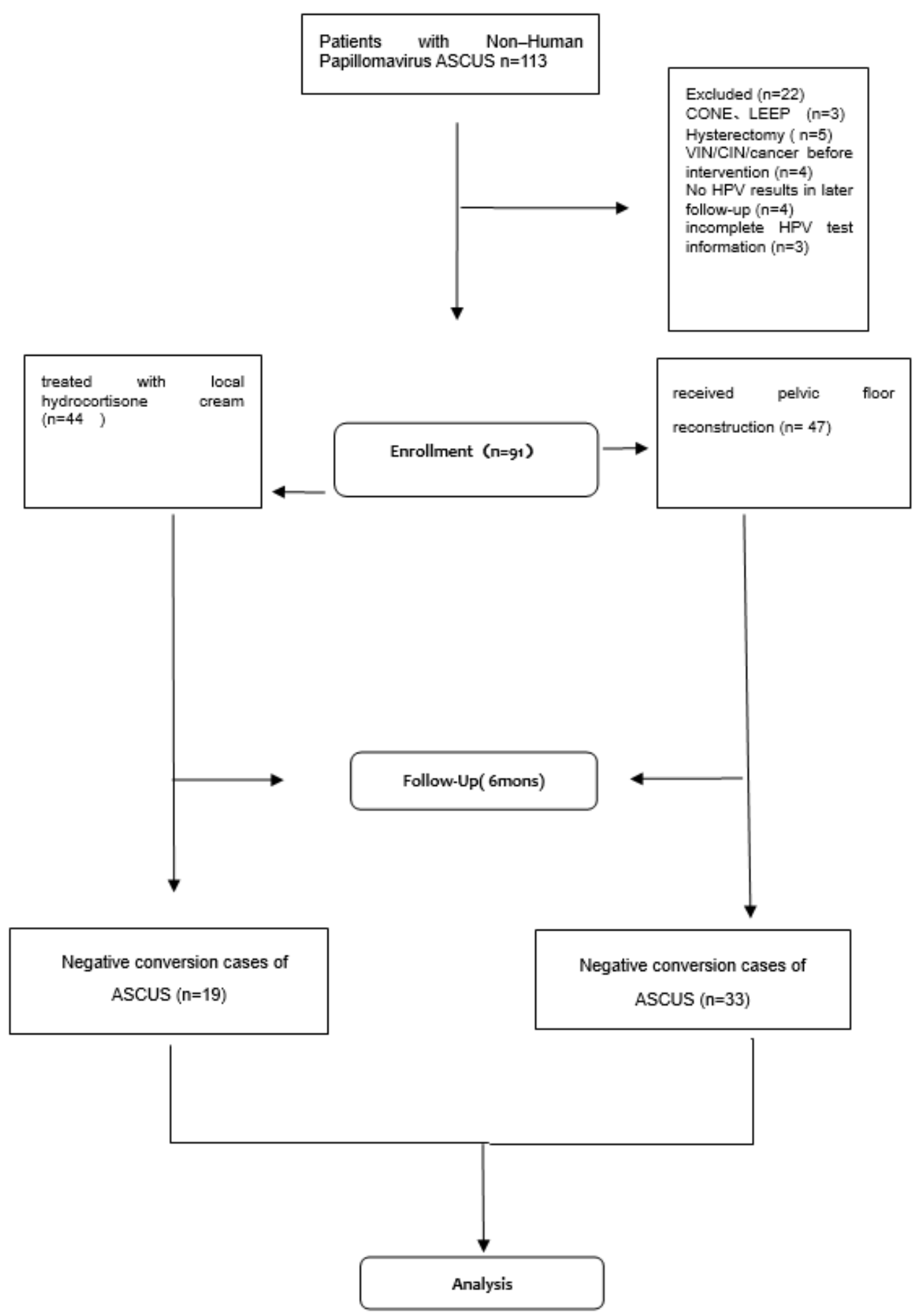

Figure 1

Flowchart of the study

\section{Supplementary Files}

This is a list of supplementary files associated with this preprint. Click to download. 
- STROBEchecklistv4combined.doc 